\title{
Pierre LASSAVE (dir.), « Cinq vies de recherche. À la naissance des Archives ", Archives des sciences sociales des religions, Hors-Série
}

Claude Langlois

\section{OpenEdition} Journals

Édition électronique

URL : https://journals.openedition.org/ress/7200

DOI : $10.4000 /$ ress. 7200

ISBN : 1663-4446

ISSN : $1663-4446$

Éditeur

Librairie Droz

Édition imprimée

Date de publication : 21 mai 2021

Pagination : 253-257

ISSN : 0048-8046

Référence électronique

Claude Langlois, "Pierre LASSAVE (dir.), "Cinq vies de recherche. À la naissance des Archives », Archives des sciences sociales des religions, Hors-Série », Revue européenne des sciences sociales [En ligne], 59-1 | 2021, mis en ligne le 21 mai 2021, consulté le 25 mai 2021. URL : http:// journals.openedition.org/ress/7200; DOI : https://doi.org/10.4000/ress.7200

Ce document a été généré automatiquement le 25 mai 2021

(C) Librairie Droz 


\title{
Pierre LASSAVE (dir.), « Cinq vies de recherche. À la naissance des Archives ", Archives des sciences sociales des religions, Hors-Série
}

\author{
Claude Langlois
}

\section{RÉFÉRENCE}

Pierre LASSAVE (dir.), 2020, « Cinq vies de recherche. À la naissance des Archives », Archives des sciences sociales des religions, Hors-Série, Paris, Éditions de l'EHESS, 228 p.

1 Ce bel ouvrage résulte d'une écriture à plusieurs mains : autant d'auteurs (André Mary, Pierre Lassave, Yvon Tranvouez, Céline Béraud, Danièle Hervieu-Léger) que de vies à raconter, présentées en autant d'abondants chapitres. Les voilà donc une ultime fois rassemblés, presque tous selon leur âge, en réalité selon leur incorporation dans le groupe: Henri Desroche (1914-1994), François-André Isambert (1924-2017), Émile Poulat (1920-2014), Jacques Maître (1925-2013), Jean-Séguy (1925-2007). Chaque biographe, libre de son approche, devait néanmoins se plier à des normes communes. L'entreprise, longuement murie sous la patiente direction de Pierre Lassave, à qui l'on doit aussi une très riche introduction (p.11-40), a abouti à la fabrication d'un livre choral très original même s'il prend, par commodité éditoriale, la forme d'un numéro hors-série de la revue Archives de sciences sociales des religions. Il en a notamment la typographie un peu serrée, mais il a bénéficié d'une iconographie inédite (26 documents photographiques) et d'un précieux index des noms propres dont sont toutefois exclus les cinq portraiturés et leur introducteur, Gabriel Le Bras. Un regret, l'absence d'une chronologie commune.

2 On connaît la saga fondatrice, quasi légendaire, racontée par les intéressés eux-mêmes au gré des anniversaires de la revue. Ils étaient quatre, sous la bienveillante protection 
de Gabriel Le Bas, qui fondèrent en 1954 le "Groupe de sociologie des religions ", avec un cinquième recruté en 1960, Jean Séguy; il se dotèrent, en 1956, d'une revue, les Archives de sociologie des religions, qu'ils rendirent vite célèbre, elle-même devenue en 1973 les Archives de sciences sociales des religions. Où donc est la nouveauté ? La démarche qui a présidé à ce livre, explique Denis Pelletier dans son avant-propos, est liée «à ce qui est au cœur de tout désir d'histoire, le dépôt d'archives » (p. 7). Leur conservation a été en effet une priorité, lourde d'investissements personnels de plusieurs auteurs. Si Émile Poulat avait décidé de déposer ses archives à l'Institut d'études avancées de Nantes, les fonds des quatre autres, selon des cheminements encore non totalement stabilisés, ont vocation à rejoindre le Grand Équipement documentaire prévu sur le campus Condorcet (Aubervilliers).

3 Une particularité souvent rappelée réunissait ce groupe des cinq: aucun n'était sociologue de formation, sinon un peu Desroche, enquêteur à Économie et Humanisme. Il en allait de même d'ailleurs pour la sociologie universitaire, refondée en 1946 par la création, au sein du CNRS, du Centre d'études sociologiques. Desroche et Poulat ont fait des études de philosophie, plutôt scolastique, et de théologie: le premier comme dominicain - alors Henri Desroches -, le second comme prêtre séculier. Le premier a quitté son Ordre lors de la crise du progressisme (1950), l'autre n'a plus figuré dans l'ordo de son diocèse (Paris), après la condamnation des prêtres ouvriers (1953-1954). Jean Séguy, entré chez les jésuites, aurait, lui, voulu y demeurer. Jacques Maître fut élevé par un oncle curé qui l'a fortement marqué, et François-André Isambert envisagea, un temps bref, le sacerdoce. Sauf Séguy, à l'apolitisme viscéral, les autres ont appartenu à la mouvance du progressisme catholique, qui se transforma parfois en une proximité avec le Parti communiste. Deux seulement, Desroche et Poulat, figurent dans «Le Maitron », le prolifique dictionnaire du monde ouvrier.

4 La guerre les a tous marqué, mais différemment. Desroche, coincé comme tant d'autres militaires en 1940 dans la poche de Dunkerque, s'en échappa par l'Angleterre et est revenu immédiatement en France. Poulat, séminariste parisien, contourna l'obligation du STO (1942) en se réfugiant dans un collège haut-savoyard et en se vieillissant de deux années. Séguy, alors en Algérie, grossit les rangs de la Première Armée française. Isambert fit de même, après avoir participé à la résistance étudiante à Paris. Maître prit les armes lors de l'insurrection dans la capitale puis s'engagea dans la $2^{\mathrm{e}} \mathrm{DB}$. Tous, sauf Séguy, se marièrent et formèrent des couples stables. Viviane Isambert-Jamati et Odile Maître menèrent, en même temps que leur mari, une carrière indépendante, la première comme brillante sociologue de l'éducation, la seconde comme médecin pédiatre.

Il ne faut toutefois point croire que l'aventure des Archives fût un long fleuve tranquille. D'abord, comme la recherche était alors purement individuelle, chacun des cinq constitua, avec des assistants personnels, son pré carré, source de difficiles délimitations de territoire. Dans la foulée, ils se sont vite trouvés en compétition pour obtenir une reconnaissance institutionnelle comme directeurs d'études à la $\mathrm{VI}^{\mathrm{e}}$ section de l'EPHE, devenue bientôt l'EHESS. Trois accédèrent à cette possibilité de dispenser un enseignement officiel, Desroche (1958), Poulat (à partir de 1963) et Isambert (1970), pour succéder à Le Bras. Maître, investi largement dans le syndicalisme, n'avait pas, semble-t-il, de telles ambitions. Séguy par contre a ressenti comme une injustice cette non élection. 
6 La revue seule les réunissait. Pourtant, au moment où celle-ci allait célébrer ses noces d'argent (25 ans), trois de ses fondateurs envisageaient de mettre un terme à leur commune aventure. Henri Desroche fit savoir en 1980 qu'il s'investissait tout entier dans un mouvement associatif dans lequel il s'était fortement engagé en même temps qu'il animait le Groupe. Ce retrait ne fit toutefois pas problème s'agissant d'une personnalité charismatique à laquelle les Archives devaient beaucoup, notamment pour avoir été « par le levier des messianismes [...] à l'écoute des religions du monde et des turbulences du Tiers-Monde » (p. 42). Il en alla différemment quand François-André Isambert, dès 1979, avait transformé son enseignement de sociologie religieuse à l'EHESS en sociologie de l'éthique et avait suscité l'essor d'une équipe dissidente vouée à cette nouvelle thématique, après avoir effectué une sérieuse initiation aux États-Unis. Jacques Maître, plus tôt encore, dès 1976, avait participé à la fondation de la sociologie médicale et s'était fait mettre à la disposition de l'INSERM. Ces trois changements d'orientation auraient pu sonner le glas d'une expérience commune, à partir des mêmes désirs d'innovation qui avaient, un quart de siècle plus tôt, permis la fondation de la revue.

7 Pourtant le Groupe de sociologie des religions a survécu à ces forces centrifuges. D'abord parce que Poulat et Séguy ont gardé la vieille maison et se sont pleinement investis dans la revue : de fait, ils sont les seuls à avoir dépassé le millier de signatures, durant le deuxième $\mathrm{XX}^{\mathrm{e}}$ siècle, pour des articles et surtout d'abondants comptes rendus. Parce que Maître, aussi, n'a jamais coupé les ponts. Et parce que ces trois-là avaient peut-être aussi leur jardin secret. Poulat s'est intéressé longuement à l'ésotérisme et Séguy à la langue provençale; Maître, différemment, devint vite sensible au sort d'ethnies vietnamiennes touchées par des bombardements américains et aux conséquences de l'usage de défoliants. Ce fut même son ultime terrain durant les dernières années de sa vie.

8 L'entreprise a également survécu parce que la revue s'était progressivement étoffée. Constant Hamès, recruté en 1965 comme collaborateur technique de Maître, devint, à partir de 1966, la plume de la revue sur l'Islam. Après 1968, le groupe bénéficia de nouveaux recrutements : un chercheur déjà confirmé, Jean-Pierre Deconchy, apporta sa compétence en psychologie expérimentale des religions et fut très actif de 1969 à 1991 ; deux jeunes femmes entrèrent alors dans le groupe : en 1972, Régine Azria, spécialiste du judaïsme, et, en 1974, Danièle Hervieu-Léger, attentive alors aux nouveaux mouvements religieux. Mais les Archives, on l'oublie souvent, ont tôt bénéficié de la présence de spécialistes extérieurs dont la fidélité et la compétence ont beaucoup compté dans son succès. Ainsi, dès 1956, de Jean Hadot (antiquité chrétienne et Judaïsme) et, dès 1961, de Jean-Marie Mayeur (historiographie allemande et italienne) ; puis, en 1968, de la génération des fondateurs, avec Doris Bensimon (judaïsme) et surtout en 1973, Françoise Aubin (religions de l'Asie).

9 Pour cinq vies donc, cinq auteurs différents. Il faut mettre à part Pierre Lassave, sociologue aux intérêts successifs (littérature, mondes urbains) dont les religions ont été le dernier et plus fécond terrain, initiateur du projet et présentateur de FrançoisAndré Isambert, qu'il a aussi accompagné dans ses dernières années. Et, pour une autre raison, Céline Béraud, la benjamine, qui présenta Jacques Maître qu'elle ne connaissait que par ses publications. Les trois autres sont, quoique de manière différentes, des proches. Desroche fut plutôt pour l'anthropologue André Mary, un initiateur à son terrain africain ; Yvon Tranvouez est un historien qui se revendique comme disciple de 
Poulat; Danièle Hervieu-Léger ne cache pas sa proximité intellectuelle et filiale avec Jean Séguy qui en fit son exécutrice testamentaire. Cette endogamie est accentuée par les fonctions de Danièle Hervieu-Léger surtout, mais aussi d'André Mary et de Pierre Lassave à la direction des Archives.

L'approche biographique a pris l'habitude de traquer les blessures cachées. Les titres énigmatiques de quatre des cinq biographies laissent entendre l'existence de telles cicatrices non refermées : Desroche (« Le mal de la frontière »), Isambert ( « Jointures et brisures »), Poulat («La marge et l'implication») et enfin Séguy («Le compromis impossible »). Plus précisément, André Mary examine longuement la mue douloureuse du Père Henri Desroches dominicain en Henri Desroche chercheur au CNRS. Danièle Hervieu-Léger accorde une large place à cette vocation refusée par la Compagnie de Jésus d'un Séguy comme à contre-emploi, puisque en quête d'ascétisme, voire de mystique. Tranvouez insiste sur cet enfant longtemps caché de Poulat, sa thèse de théologie soutenue en 1950 à Fribourg-en-Brisgau. Lassave a expliqué comment la vie d'Isambert fut traversée d'épisodes cyclothymiques devenus très handicapants pour lui, rendant aussi ses soudaines absences difficiles à gérer par ses entourages.

11 S'en tenir là toutefois pourrait laisser croire que l'essentiel, l'œuvre de chacun, n'aurait pas été examinée à nouveaux frais. Tout au contraire. Et avec un triple avantage sur les précédentes présentations plus ou moins auto-légitimatrices. D'abord, il s'agit d'une expertise extérieure, quelle que soit la proximité des auteurs et autrices, apportée par le regard d'une autre génération. Ensuite, les disparitions, souvent récentes, des intéressés ont permis la prise en compte de la totalité d'une vie, démarche qui fait mieux apparaître, à côté de précocités connues, la reconversion réussie d'Isambert comme initiateur de la sociologie de l'éthique et la fécondité tardive de Maître dont l'œuvre principale éclot soudain au lendemain même de sa retraite. Reste surtout la possibilité de croiser ces cinq trajectoires scientifiques, ce qui permet de mieux délimiter le champ des possibles, de comparer les intérêts pour la revue avec les lourds investissements personnels et aussi de montrer le rapport différent de chacun entre production scientifique et modélisation sociologique.

12 Je voudrais terminer cette présentation sur l'aveu d'un potentiel conflit d'intérêt. Non seulement à cause de ma proximité avec la plupart des auteurs, mais plus encore avec chacun des cinq fondateurs. J'ai rêvé après 1968 des dieux de Desroche; j'ai suivi le séminaire de Poulat à partir de 1970 ; j'ai partagé les analyses d'Isambert sur la religion populaire ; j'ai été suffisamment proche de Séguy pour lui demander d'être à mon jury de thèse sur les congrégations religieuses ; et je me suis rapproché, plus tard, de Maître par un commun et durable intérêt pour Thérèse de l'Enfant-Jésus. Sans doute, suis-je, à ces titres divers, parmi les historiens français de ma génération, celui qui leur fut proche si longuement. Cet aveu en lui-même n'aurait pas grand intérêt s'il ne permettait aussi de laisser pressentir, au-delà des relations interpersonnelles, les rapprochements fructueux entre disciplines, mais aussi les géographies d'influence entre Italie et Québec. Il faudrait aussi évoquer ce qui ne fut pas, comme les évitements de la psychanalyse lacanienne et surtout de la sociologie de Bourdieu, malgré un tardif dialogue suscité par Maître avec le sociologue du Collège de France.

Ces biographies croisées des refondateurs de la sociologie des religions en France au lendemain de la Seconde Guerre mondiale restituent la part des individus - un masculin alors commun - là où la revue imposait une référence collective. Elles montrent aussi la fécondité de la matrice catholique, clercs comme laïcs, dont 
l'historiographie n'a pas encore pris l'entière mesure, au regard de la filière marxiste, beaucoup mieux connue. Dans les années 1990, le Groupe de sociologie des religions, de par la volonté du CNRS qui ne voulait plus d'équipes qui ne se rattachaient pas à une université ou à un grand établissement, s'est scindé en deux mais la revue est demeurée une et l'existence de ces deux équipes a paradoxalement conduit à mettre davantage en évidence le triangle institutionnel privilégié des sciences sociales des religions : CNRS, EHESS, EPHE. Comment mesurer le succès des cinq, sinon par l'existence d'une revue, les Archives, qui demeure un lieu unique en francophonie de confrontation des sciences sociales des religions et de réflexion sur leurs perspectives méthodologiques?

\section{AUTEURS}

\section{CLAUDE LANGLOIS}

Paris, EPHE - GSRL 